\section{Análise da distribuição espacial da mortalidade neonatal e de fatores associados, em Salvador, Bahia, Brasil, no período 2000-2006}

\author{
Spatial analysis of neonatal mortality and \\ associated factors in Salvador, Bahia \\ State, Brazil, 2000-2006
}

\author{
1 Escola de Enfermagem, \\ Universidade Federal do Rio \\ Grande do Sul, Porto Alegre, \\ Brasil. \\ 2 Instituto de Saúde Coletiva \\ Universidade Federal da \\ Bahia, Salvador, Brasil. \\ 3 Escola Nacional de Saúde \\ Pública Sergio Arouca, \\ Fundação Oswaldo Cruz, Rio \\ de Janeiro, Brasil. \\ 4 Centro Biomédico, \\ Universidade do Estado do \\ Rio de Janeiro, Rio de Janeiro, \\ Brasil. \\ Correspondência \\ M. C. N. Costa \\ Instituto de Saúde Coletiva, \\ Universidade Federal da \\ Bahia. \\ Rua Basílio da Gama s/n, \\ Salvador, $B A$ \\ 40110-040, Brasil. \\ mcncosta@ufba.br
}

\section{Abstract}

An ecological study was performed to identify patterns in the spatial distribution of neonatal mortality and risk areas as well as associated factors in Salvador, Bahia State, Brazil, 2000-2006. A thematic map was constructed, and techniques were used for formal assessment of spatial dependence. No spatial and spatial regression models were used to assess possible relationships between selected factors and spatial distribution of neonatal mortality. Spatial autocorrelation was observed in neonatal mortality $(I=0.17$; $p=0.0100$ ), so its distribution was non-random. The analysis showed a spatial pattern in which the highest risk (> 9.0/1,000 live births) was concentrated in the downtown areas and peripheral neighborhoods with the highest concentration of low-income families, and this was associated with the target risk factors. The proportion of low birth weight newborns was the only variable that was significantly associated with neonatal mortality. Living conditions may have contributed to the uneven spatial distribution of neonatal mortality in this city.

Spatial Analysis; Neonatal Mortality (Public Health); Risk Zone; Risk Factors
Annelise C. Gonçalves 1

Maria da Conceição Nascimento Costa ${ }^{2}$

José Uéleres Braga 3,4

\section{Introdução}

O desenvolvimento das técnicas de análise estatística espacial, apoiadas pelos Sistemas de Informações Geográficas (SIG), vem representando um valioso aporte para a Epidemiologia, na medida em que amplia a sua capacidade para identificar grupos populacionais sob maior risco de adoecer e morrer, bem como fatores explicativos da ocorrência destes eventos em populações.

A pertinência da aplicação dessas ferramentas decorre do fato de o espaço geográfico se constituir em uma dimensão dinâmica, resultante de interações complexas e temporais entre os diversos fatores que definem a sua organização, além de se constituir em um componente intrínseco dos determinantes das condições de vida ${ }^{1}$. Revestido dessas características, o espaço vem sendo utilizado como um critério que tem se mostrado bastante útil no estudo da dinâmica e perfil epidemiológico das doenças e agravos à saúde 2 e, sobretudo, das desigualdades sociais na sua distribuição.

Em um estudo aplicando o SIG, Loyola et al. ${ }^{3}$ observaram que, entre 1995 e 2000, o risco de morte infantil em países menos desenvolvidos da região das Américas era 20 vezes maior que naqueles mais desenvolvidos. Agrupamentos espaciais com altas taxas de mortalidade infantil em áreas socialmente marginalizadas foram identificados em uma cidade mexicana, nos anos de 2000-2003, mediante análise utilizando essa 
mesma estratégia ${ }^{4}$. Igualmente, investigação conduzida no Estado do Michigan, Estado Unidos, de 2004-2006, possibilitou a demonstração de áreas de risco para baixo peso ao nascer, um dos mais importantes fatores de risco para morte neonatal 5. Para os autores desses estudos, o uso adequado dessa tecnologia torna mais fácil a identificação de áreas que necessitam de maior atenção das políticas e programas de saúde, e a produção de informações relevantes para subsidiar o planejamento de intervenções específicas para as áreas de risco, contribuindo para a instituição de ações de saúde pública mais equânimes e efetivas.

Especificamente no que diz respeito à mortalidade neonatal, os estudos que fazem uso da análise espacial também têm fornecido informações valiosas sobre áreas e grupos sociais prioritários 6,7,8, sinalizando para a necessidade de implementação de programas voltados para prevenção e controle de mortes infantis precoces. A influência de fatores individuais e coletivos como pobreza e privação social nos níveis da mortalidade neonatal foi evidenciada por estudo desta natureza desenvolvido no Município do Rio de Janeiro ${ }^{9}$. Em bairros de Goiânia, Goiás, onde residia a população de baixa renda, foram observadas as mais elevadas taxas de mortalidade neonatal 6. Por sua vez, achados conflitantes foram encontrados no Estado do Paraná, de 19972001, onde tanto os aglomerados de municípios de melhor como os de pior situação socioeconômica apresentaram as mais baixas taxas dessa mortalidade e, o mais surpreendente, com valores semelhantes. Esse resultado poderia indicar a contribuição de outros fatores, além dos socioeconômicos, a exemplo dos efeitos dos serviços de saúde, demonstrando a atuação destes últimos na intermediação entre as condições de vida e a situação de saúde 10.

Outro aporte da análise espacial é possibilitar a identificação de dependência espacial ou autocorrelação espacial, que indica alta probabilidade de similaridade entre taxas de adoecimento ou mortes em áreas geográficas adjacentes, ou seja, informa a não independência dessas taxas às suas posições relativas no espaço. Exemplos dessa contribuição no estudo da mortalidade neonatal podem ser observados nos estudos conduzidos por Nascimento et al. 11, Moraes Neto et al. 6 e Leal \& Szwarcwald 12.

Vale salientar que, no Brasil, ainda é alto o risco de morte entre os recém-nascidos durante os primeiros 28 dias de vida. A taxa de mortalidade neonatal nesse país (Departamento de Informática do SUS. Indicadores e dados básicos - Brasil 2009. http://w3.datasus.gov/datasus/datasus. php, acessado em 17/Jul/2009), em 2007, era de
10,7/1.000 nascidos vivos, ou seja, aproximadamente 2,5 vezes superior à média dos Estados Unidos (4/1.000 nascidos vivos) em 2006 e, cerca de 10 vezes maior que a do Japão (1/1.000 nascidos vivos), em 2004 13, dados estes sugestivos de que muitas mortes ocorridas no Brasil no período neonatal poderiam ter sido evitadas. Ainda em 2007, em Salvador, capital da Bahia, estado situado no nordeste brasileiro, uma das regiões mais pobres do país, essa mortalidade era de 15,4/1.000 nascidos vivos, enquanto que em Curitiba (Paraná) e Florianópolis (Santa Catarina), capitais localizadas na Região Sul, de maior desenvolvimento socioeconômico, as taxas de mortalidade neonatal já eram inferiores a 10/1.000 nascidos vivos desde o final dos anos de 1990 (http://w3.datasus.gov/datasus/datasus. php, acessado em 17/Jul/2009). Esse cenário evidencia a necessidade de envidar esforços no sentido de identificar os fatores mais prevalentes dentre aqueles que contribuem para determinar tal situação, no propósito de subsidiar os gestores da área de saúde no planejamento de ações voltadas para a sua reversão.

Os determinantes da mortalidade neonatal são múltiplos, complexos, e se relacionam à interação de variáveis biológicas (maternas e do recém-nascido), assistenciais e socioeconômicas 14, variando conforme diferentes contextos, tempo e lugar. Essa dinâmica torna imperativa a realização de investigações que, a exemplo de análises espaciais, sejam capazes de identificar áreas e grupos sociais de maior risco e fornecer elementos para ampliar a compreensão da ocorrência da doença e morte, especialmente em países em desenvolvimento. Nessa perspectiva, este estudo teve como objetivos identificar padrões na distribuição espacial e áreas de risco para a mortalidade neonatal, bem como, examinar possíveis associações entre fatores relativos à atenção à saúde materno-infantil, características maternas e condições de nascimento, e a variação espacial desta mortalidade, em Salvador, Bahia, no período de 2000-2006.

\section{Material e métodos}

Realizou-se um estudo de agregados espaciais, tendo como unidade de análise a Zona de Informação (ZI). Esta corresponde a áreas $(n=93)$ em que a cidade de Salvador foi subdividida pela Companhia de Desenvolvimento da Região Metropolitana (CONDER), baseada em critérios socioeconômicos, de infraestrutura físicoadministrativa e planejamento, e de compatibilização com os setores censitários do Instituto Brasileiro de Geografia e Estatística (Cidades@. 
http://www.ibge.gov.br/cidades/default, acessado em 10/Nov/2011).

Os dados sobre óbitos e nascidos vivos, ocorridos entre 2000 e 2006, foram obtidos do Departamento de Informação de Saúde da Secretaria de Saúde daquele município, que forneceu cópia dos respectivos bancos de dados (SIM e SINASC) em meio magnético (CD-ROM) 15. Para o georreferenciamento dos endereços dos óbitos e nascimentos utilizou-se o LOCALIZA, programa desenvolvido no Instituto de Saúde Coletiva, Universidade Federal da Bahia (Evangelista D. Sistema de Georreferenciamento de Endereços em Unidades Territoriais, Salvador, Brasil). Os endereços que esse programa não conseguiu georreferenciar foram classificados e codificados manualmente, com o auxílio de mapas cartográficos e do aplicativo Google Earth (http://www. google.com.br/intl/pt-BR/earth/index.html), via Internet. Em virtude da grande quantidade de endereços não registrados na Declaração de Óbitos ocorridos nos anos de 2000-2002, procedeuse o relacionamento não probabilístico com bancos de dados de nascidos vivos dos respectivos anos, utilizando como variáveis de pareamento a data de nascimento, o nome da mãe e, quando disponível, o peso do recém-nascido.

A CONDER forneceu as malhas digitais das ZI (bases cartográficas do município) em formato shapefile (shp). Dos bancos de dados de nascidos vivos foram levantados e distribuídos por ZI os seguintes indicadores: características maternas (proporção de mães com escolaridade inadequada à idade, proporção de nascidos vivos de mães adolescentes de 10-19 anos, e proporção de nascidos vivos de mães com idade igual ou superior a 35 anos); condições de nascimento (proporção de nascidos vivos com peso inferior a $2.500 \mathrm{~g}$ e proporção de nascidos vivos com idade gestacional abaixo de 37 semanas); atenção à saúde materno-infantil (proporção de nascidos vivos de mães com número de consultas pré-natais inadequado à idade gestacional, proporção de nascidos vivos de partos cesáreos, e proporção de nascimentos vivos em hospitais públicos). "Proporção de mães com escolaridade inadequada à idade" e "proporção de nascidos vivos de mães com número de consultas pré-natais inadequado à idade gestacional” foram calculadas conforme preconizado pelo Ministério da Educação para escolarização e número de consultas recomendado pelo Ministério da Saúde 16 para cada trimestre da gestação, respectivamente. Devido aos altos percentuais de não registro do Apgar (média de preenchimento anual de apenas 22\% nos anos de 2000-2004), não foi possível avaliar este índice como um dos fatores associados à mortalidade neonatal, apesar de sua reconheci- da importância como indicador das condições de nascimento e qualidade de atenção ao recémnascido em sala de parto ${ }^{17}$.

Em função do pequeno número de óbitos neonatais e de nascidos vivos em algumas ZI, a análise dos dados foi realizada para 2000-2006, como um todo, obtendo-se taxas médias, calculadas com base na divisão do somatório do número de óbitos neonatais ocorrido nos sete anos do estudo em cada ZI pelo somatório do número de nascidos vivos no mesmo período na ZI correspondente, multiplicado por mil. Para identificar padrões na distribuição espacial da mortalidade neonatal, foi realizada análise exploratória mediante inspeção visual de mapas temáticos construídos com o emprego do programa ArcView 3.3 (Environmental Systems Research Institute Inc., http://www.esri.com/software/arcview/) e do módulo de estatística espacial do S-PLUS 6.0 (Mathsoft Inc., Seattle, Estados Unidos), a partir das taxas de mortalidade neonatal das ZI, brutas e após transformação do tipo Freeman-Tukey 18. Essa transformação, além de minimizar as variações decorrentes do pequeno número de óbitos e nascimentos em algumas ZI e possibilitar a identificação de padrões espaciais, permite verificar relações mediadas pelo espaço e não induzir a autocorrelação espacial. Para obtenção da matriz de vizinhança por adjacência (vizinhos próximos com pelo menos um ponto de fronteira em comum) foram excluídas duas ZI referentes às ilhas pertencentes ao município. A seguir, avaliouse formalmente a existência de autocorrelação espacial das taxas de mortalidade neonatal das ZI utilizando-se o Índice Global de Moran 19, enquanto a identificação das áreas de risco foi realizada por meio do Índice Local de Autocorrelação Espacial de Moran (LISA/Local Indicator of Spatial Association), com nível de 0,05 de significância, usando-se o programa GeoDa (Spatial Analysis Laboratory, University of Illinois, Urbana-Champaign, Estados Unidos). O referido índice, em geral, varia de +1 a -1 e tem a vantagem de ser mais sensível a valores extremos.

A relação entre fatores de risco selecionados e mortalidade neonatal foi avaliada por meio da correlação espacial bivariada (Índice de Moran bivariado). Tendo em vista a identificação de autocorrelação espacial dos resíduos, aplicou-se modelos de regressão linear espacial SAR (Spatial Auto Regressive), sendo a taxa transformada de mortalidade neonatal a variável dependente e os fatores anteriormente informados, as independentes. Análise de regressão não espacial também foi realizada visando a demonstrar a vantagem do uso de modelos espaciais nesses tipos de estudo por apresentarem resultados mais precisos. 
O protocolo desta pesquisa foi aprovado pelo Comitê de Ética em Pesquisa do Instituto de Saúde Coletiva da Universidade Federal da Bahia, sob registro CEP: 012-09/CEP-ISC.

\section{Resultados}

No período de 2000-2006, foram registrados 271.840 nascimentos vivos de mães residentes em Salvador e 5.097 óbitos neonatais. Apenas $8 \%$ desses óbitos e $0,7 \%$ dos nascimentos não foram georreferenciados devido a endereços não registrados ou incompletos e a inexistência do logradouro nos cadastros dos programas e ferramentas utilizados. A taxa média de mortalidade neonatal do município, para o período do estudo, calculada baseando-se nos dados georreferenciados foi de 17,4/1.000 nascidos vivos.

A distribuição da mortalidade neonatal nas ZI da cidade, no período 2000-2006, apresenta um padrão espacial caracterizado por áreas com as mais altas taxas transformadas $(\geq 9,0 / 1.000$ nascidos vivos), localizadas de norte a sul na região oeste da cidade, e taxas intermediárias (7,6-8,9/1.000 nascidos vivos) na região centroleste, sendo mantido o mesmo padrão das taxas brutas. A Figura 1 mostra que as áreas com as menores taxas transformadas de mortalidade neonatal (3,2-5,5/1.000 nascidos vivos) foram: ZI 10 (Graça), ZI 4 (Alto da Sereia/Vila Matos), localizadas ao sul, ZI 8 (Pituba/Parque Júlio César), ZI 22 (Stiep/Armação), ZI 31-A (Bolandeira), localizadas à leste, ZI 38-A (Caminho de Areia), na região oeste e 72 (limite com a Usina Siderúrgica da Bahia), ao norte. O Índice de Moran Global para essa distribuição foi de 0,1717 ( $p=0,0100)$.

Cotejando-se a Figura 1 (que identifica a localização das ZI de Salvador) com a Figura 2 (que ilustra o padrão espacial das taxas transformadas da mortalidade neonatal), observa-se sete ZI (23, 23A, 24, 24D, 32, 33 e 39) agrupadas com taxas igualmente altas (alto/alto) localizadas ao sudoeste, em áreas do centro do município, com valores iguais ou acima de 7,6/1.000 nascidos vivos. Dois aglomerados (ZI 61 e 1), um localizado na região nordeste e outro na região sul do município, apresentam taxas um pouco mais baixas (baixo/baixo) e representam locais de baixo risco para óbito neonatal. Grupamentos considerados de transição, foram formados por ZI de taxas baixas com áreas vizinhas com valores mais altos (baixo/alto), situadas no centro (ZI 15B, 24A, 24C) e ao norte (ZI 72) e aqueles constituídos por ZI com taxas mais altas, mas com vizinhas com baixas taxas (alto/baixo), situadas ao sul da cidade (ZI 9) e na região leste (ZI 55) (Figura 3).
Valores elevados do percentual de nascimentos vivos em hospital público concentraram-se na porção central, em direção ao norte da cidade, e os mais baixos no sul e leste. Padrão semelhante foi observado na distribuição dos nascidos vivos de mães com escolaridade inadequada à idade e daqueles de mães adolescentes. Já as proporções de nascimentos vivos de mães com idade igual ou superior a 35 anos e de parto cesáreo mostraram um padrão espacial inverso, com maiores valores em ZI situadas ao sul e ao leste do município. As proporções de nascidos vivos prematuros e de baixo peso apresentaram distribuição mais homogênea, com valores mais elevados na direção norte-sul. Grande número de ZI, localizadas na região central e ao norte do município, apresentou proporções de nascidos vivos de mães com pré-natal inadequado à idade gestacional.

Os resultados estatisticamente significantes dos coeficientes I de Moran da taxa transformada na análise bivariada com os fatores estudados, apresentados na Tabela 1, indicam a presença de correlação espacial entre mortalidade neonatal e proporção de nascimentos em hospital público ( $\mathrm{I}=0,15 ; \mathrm{p}=0,0300$ ), proporção de mães com escolaridade inadequada à idade $(\mathrm{I}=0,17$; $\mathrm{p}=0,0400$ ), proporção de nascidos vivos de mães adolescentes ( $\mathrm{I}=0,17 ; \mathrm{p}=0,0300)$, proporção de nascidos vivos com peso inferior a $2.500 \mathrm{~g}$ $(\mathrm{I}=0,19 ; \mathrm{p}=0,0300)$ e proporção de nascidos vivos de mães com número de consultas prénatais inadequado à idade gestacional $(I=0,21$; $\mathrm{p}=0,0100$ ), tendo estes dois últimos fatores os maiores coeficientes de correlação.

Na Tabela 2, que apresenta os coeficientes de regressão linear múltipla e de regressão linear espacial, verifica-se que a maior contribuição foi da proporção de nascidos vivos com peso inferior a $2.500 \mathrm{~g}$, observando-se que para cada aumento de uma unidade nesta proporção haverá, em média, um aumento de 0,64 (regressão não espacial) e de 0,65 (regressão espacial) na taxa desta mortalidade. Esse foi o único fator que se mostrou estatisticamente associado $(\mathrm{p}<0,0000)$ à mortalidade neonatal.

\section{Discussão}

Os resultados do presente estudo evidenciam uma distribuição bastante desigual da mortalidade neonatal no espaço urbano de Salvador, em 2000-2006, cujas taxas transformadas variaram entre 3,2/1.000 nascidos vivos e 16,1/1.000 nascidos vivos. Os maiores riscos para essa mortalidade concentraram-se em áreas deterioradas e crescimento negativo, com indicadores de saúde e de condições de vida desfavoráveis 20 , locali- 
Figura 1

Distribuição do território do Município de Salvador, Bahia, Brasil, segundo Zonas de Informação, 2000.

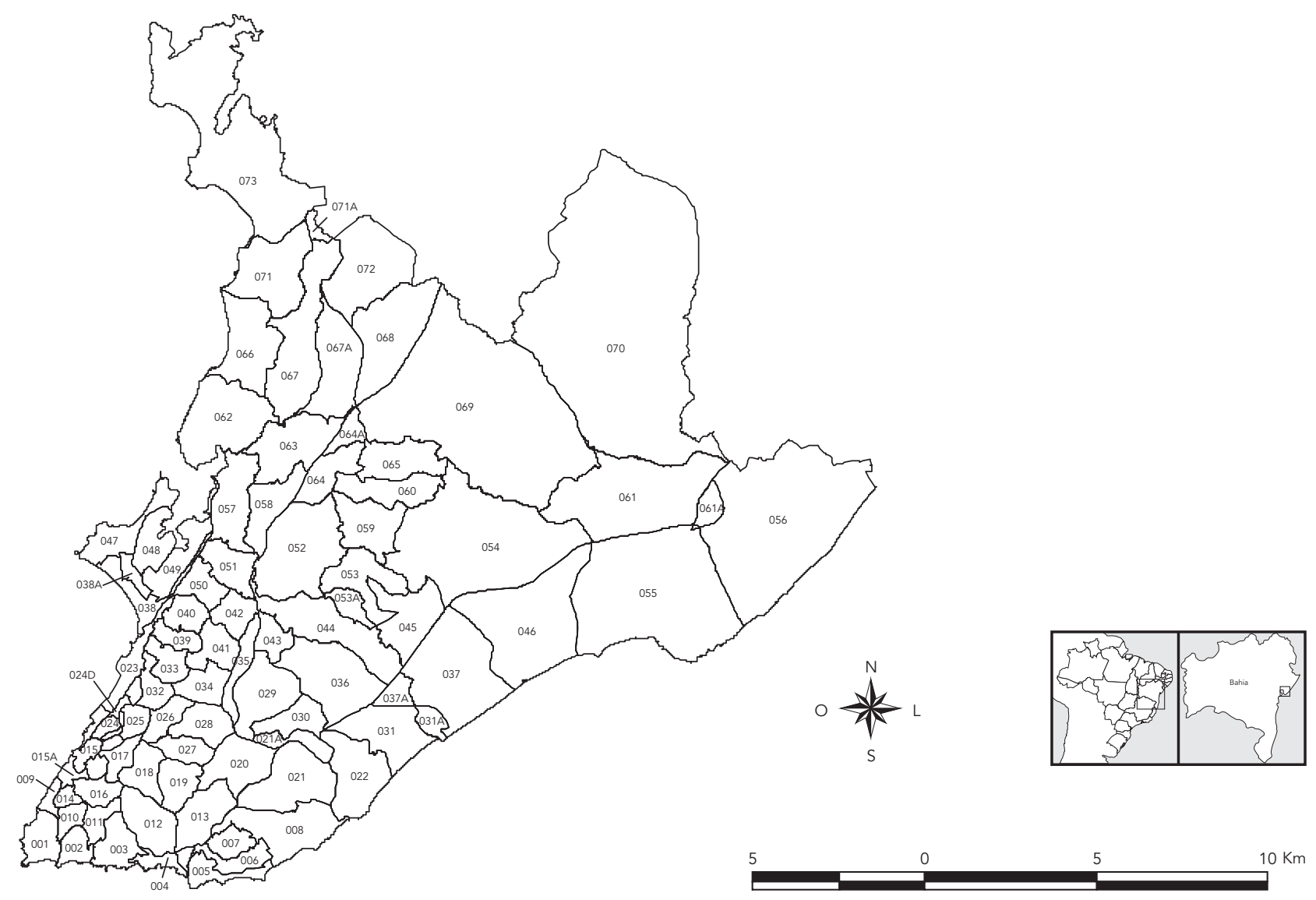

\section{Zonas de informação}

001-Barra

002-Morros do Gato e Ipiranga/Apipema 003-Ondina/Campus Universitário 004-Alto da Sereia/Vila Matos 004A-Parque Cruz Aguiar 005-Largo da Mariquita/Av. Amaralina 006-Vale das Pedrinhas 007-Nordeste de Amaralina 008-Pituba/Parque Júlio César 009-Vitória 010-Graça

011-Alto das Pombas/Federação 012-Engenho Velho da Federação 013-Horto Florestal/Candeal 014-Canela

015-São Pedro

015A-Forte de São Pedro 015B-Campo da Pólvora 016-Garcia

017-Tororó/Fonte Nova 017A-Barris

018-Engenho Velho das Brotas 019-Acupe de Brotas/Daniel Lisboa
020-Campinas de Brotas

021-Ataigara/Caminho das árvores 021A-Av. Tancredo Neves 022-STIEP/Armação 023-Água de Meninos 023A-Comércio 024-Praça Municipal/Centro 024A-Pelourinho 024C-Baixa dos Sapateiros 024D-Santo Antônio 024E-Ladeira de Santana 025-Nazaré/Saúde 026-Matatu/Santo Agostinho 027-Cosme de Farias 028-Luiz Anselmo/Vila Laura 029-Cabula

030-Pernambués

031-Boca do Rio

031A-Bolandeira 032-Barbalho/Lapinha 033-Caixa D'Água 034-Quintas/Cidade Nova 035-Retiro/Acesso Norte
036-19BC/Narandiba

037-Pituaçu

037A-Imbuí

038-Calçada/Mares/Roma

038A-Caminho de Areia

038B-Baixa do Fiscal

039-Sieiro/Japão/Pero Vaz

040-Liberdade

041-|API

042-Fazenda Grande

043-São Gonçalo do Retiro

044-Tancredo Neves/Engomadeira

045-Centro Administrativo da Bahia - CAB

046-Patamares

047-Bonfim/Ribeira

048-Massaranduba

049-Uruguai/Alagados

049A-Voluntários da Pátria/Suburbana

050-São Caetano/Alto do Peru

051-Capelinha de São Caetano

052-Mata Escura

053-Sussuarana

053A-Estrada do Beiru
054-Canabrava

055-Piatã/Itapuã

056-Aeroporto/Stella Maris

057-Lobato

058-Campinas/Marechal Rondon

059-Pau da Lima

060-Sete de Abril

061-Mussurunga/São Cristovão

062-Plataforma

063-Pirajá

064-Dom Avelar

064A-Porto Seco Pirajá

065-Castelo Branco

066-Praia Grande-Periperi

067-Parque São Bartolomeu

067A-Represa do Cobre

068-Valéria

069-Cajazeiras/Águas Claras

070-Estrada/CIA Aeroporto

071-Coutos

071A-Felicidade

072-Limite da USIBA

073-Paripe/Base Naval 
Distribuição espacial das taxas transformadas de mortalidade neonatal, segundo Zonas de Informação. Salvador, Bahia, Brasil, 2000-2006.
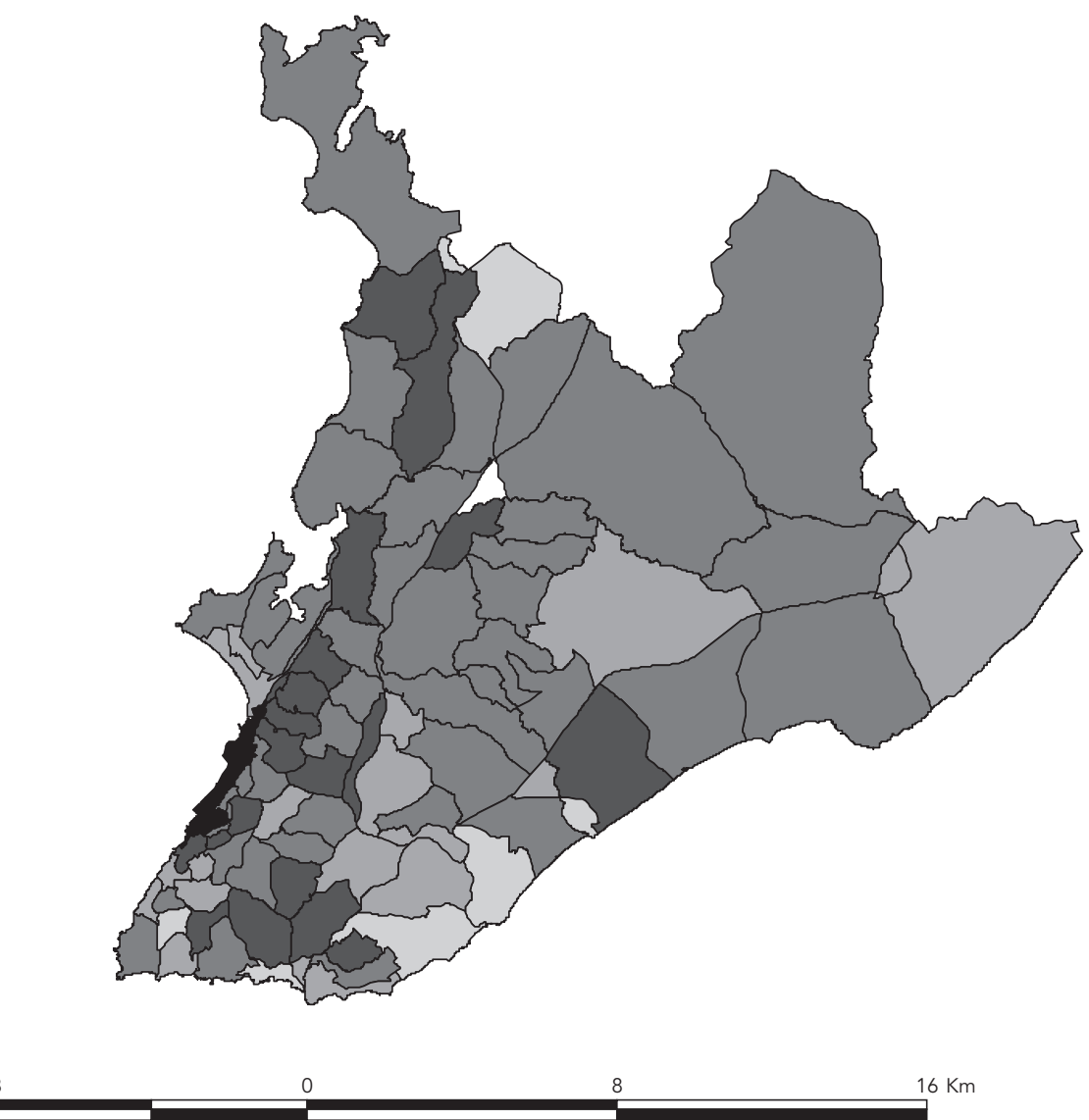

Taxa média transformada por 1.000 nascidos vivos
Sem dados
$3,2-5,5$
$5,6-7,5$
$7,6-8,9$
$9,0-10,9$
$11,0-16,1$

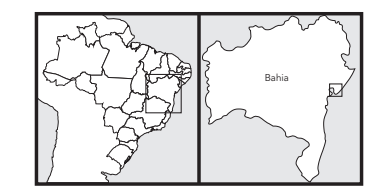

$\mathrm{S}$
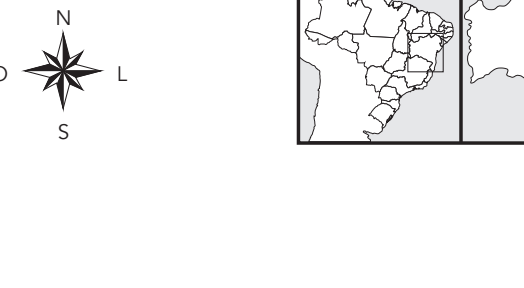

$$
\text { ( }
$$

\section{.}




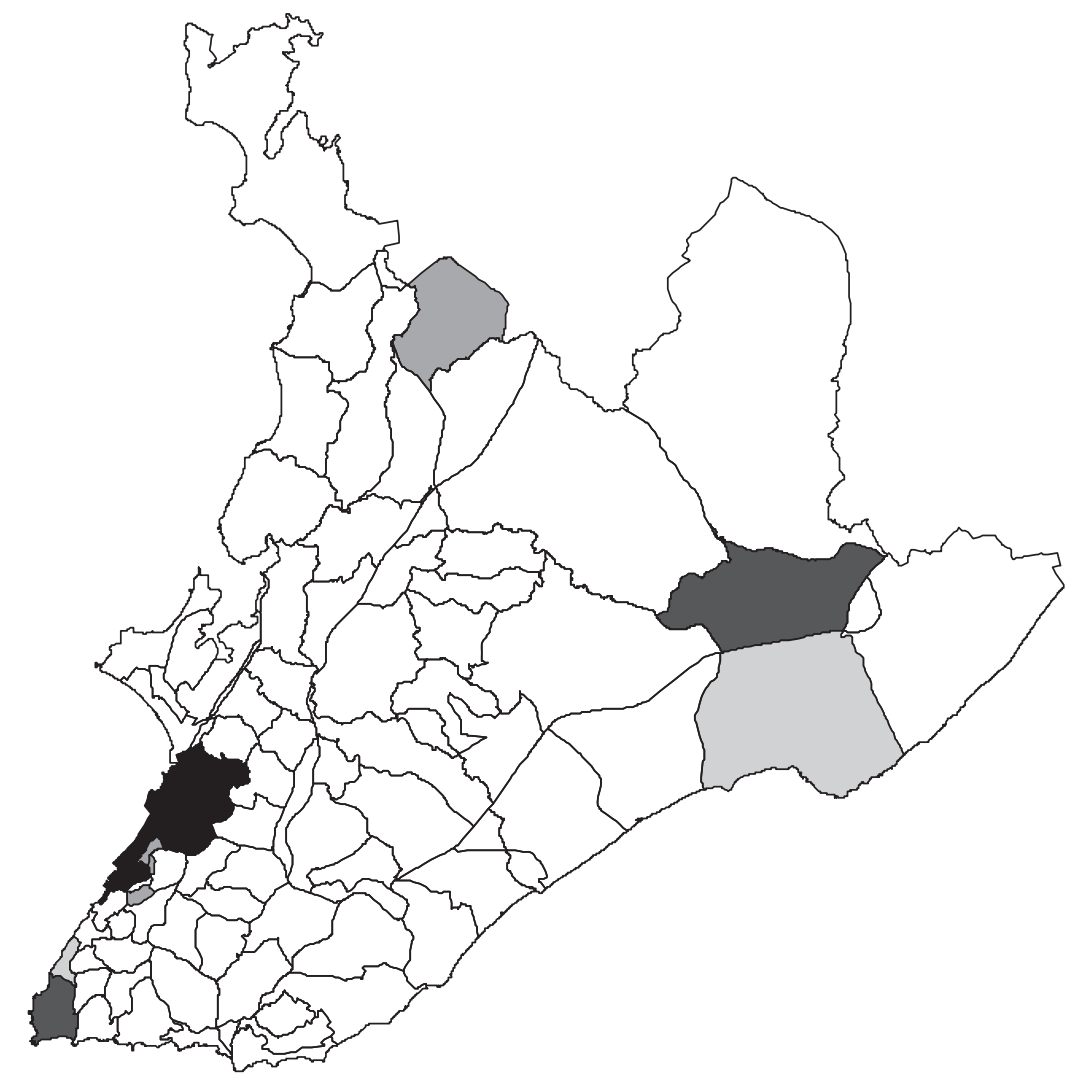

Alto/Alto
Baixo/Baixo
Baixo/Alto
Alto/Baixo
Autocorrelação espacial não significante

* Avaliada pelo índice de Moran local a 0,05.

marcada pelas deficiências de infraestrutura e de serviços básicos 22, situação que, certamente, repercute nas condições de saúde da população que ali habita e, em especial, da população infantil, com reflexo na mortalidade neonatal.

Vale referir que, em uma das zonas que compõem esse aglomerado (ZI 33 - Caixa d'Água) a assistência materno-infantil é sabidamente deficiente. Nela se encontram dois hospitais públicos com atendimento materno-infantil, mas apenas um deles possui leitos de unidade de tratamento intensivo (UTI) neonatal, e em pequeno número. Na outra zona (ZI 23 - Água de Meninos), está localizado um hospital também com pequeno 
Tabela 1

Coeficientes I de Moran bivariado para a relação entre taxa de mortalidade neonatal * e alguns indicadores relativos a características maternas, condições de nascimento e de atenção à saúde. Salvador, Bahia, Brasil, 2000-2006.

\begin{tabular}{|c|c|c|}
\hline Indicadores & I & Valor de $\mathrm{p}$ \\
\hline Proporção de nascidos vivos de mães com escolaridade inadequada à idade & 0,1661 & 0,0400 \\
\hline Proporção de nascidos vivos de mães adolescentes & 0,1650 & 0,0300 \\
\hline Proporção de nascidos vivos de mães com idade $\geq 35$ anos & 0,0484 & 0,9900 \\
\hline Proporção de nascidos vivos de parto cesáreo & 0,8830 & 0,2400 \\
\hline Proporção de nascidos vivos com idade gestacional abaixo de 37 semanas & 0,1115 & 0,3100 \\
\hline Proporção de nascidos vivos com peso inferior a $2.500 \mathrm{~g}$ & 0,1893 & 0,0300 \\
\hline Proporção de nascimentos vivos em hospital público & 0,1526 & 0,0300 \\
\hline Proporção de nascidos vivos de mães com número de consultas pré-natais inadequado à idade gestacional & 0,2110 & 0,0100 \\
\hline
\end{tabular}

* Taxa transformada Freeman-Tukey.

Tabela 2

Coeficientes de regressão linear múltipla e coeficientes de regressão linear espacial para a associação entre a taxa transformada de mortalidade neonatal * e fatores relativos às características maternas, condições de nascimento e de atenção à saúde para as Zonas de Informação. Salvador, Bahia, Brasil, $2000-2006$.

\begin{tabular}{|c|c|c|c|c|}
\hline Indicadores & $\begin{array}{l}\text { Coeficiente de regressão } \\
\text { linear não espacial (ß) }\end{array}$ & Valor de p & $\begin{array}{l}\text { Coeficiente de regressão } \\
\text { linear espacial (ß) }\end{array}$ & Valor de $\mathrm{p}$ \\
\hline $\begin{array}{l}\text { Proporção de nascidos vivos de mães com escolaridade } \\
\text { inadequada à idade }\end{array}$ & 0,0204 & 0,6867 & 0,0193 & 0,7076 \\
\hline Proporção de nascidos vivos de mães adolescentes & 0,0198 & 0,8381 & 0,2103 & 0,8154 \\
\hline $\begin{array}{l}\text { Proporção de nascidos vivos de mães com idade } \geq 35 \\
\text { anos }\end{array}$ & 0,1633 & 0,0716 & 0,1712 & 0,0683 \\
\hline $\begin{array}{l}\text { Proporção de nascidos vivos com idade gestacional < } 37 \\
\text { semanas }\end{array}$ & 0,2076 & 0,1825 & 0,2189 & 0,1853 \\
\hline Proporção de nascidos vivos com peso $<2.500 \mathrm{~g}$ & 0,6404 & 0,0000 & 0,6512 & 0,0000 \\
\hline Proporção de nascidos vivos de parto cesáreo & $-0,0448$ & 0,4046 & $-0,0454$ & 0,3961 \\
\hline Proporção de nascimentos vivos em hospital público & 0,0061 & 0,74 & 0,0059 & 0,737 \\
\hline $\begin{array}{l}\text { Proporção de nascidos vivos de mães com número de } \\
\text { consultas pré-natais inadequado à idade gestacional }\end{array}$ & $-0,0411$ & 0,4364 & 0,0398 & 0,4532 \\
\hline
\end{tabular}

* Taxa transformada Freeman-Tukey.

número de leitos obstétricos, porém sem leitos de UTI neonatal. Nas demais ZI, não existem hospitais, o que deve exigir o deslocamento da população para outras áreas da cidade que dispõem desse tipo de atenção à saúde. Porém, sabe-se que em Salvador convive-se com carência de leito de UTI, mesmo em áreas cuja população apresenta melhores condições de vida. Em se tratando da mortalidade neonatal, é necessário, não só o acesso a serviços de saúde da atenção básica (pré-natal) como também a serviços de maior complexidade e de boa qualidade para o atendimento materno-infantil de alto risco.
Salienta-se ainda que, nessas regiões de maior mortalidade neonatal, também se concentraram as maiores proporções de nascimentos vivos em hospitais públicos, de recém-nascidos de baixo peso, de mães adolescentes e de mães com escolaridade inadequada à idade e com número de consultas pré-natais inadequado à idade gestacional. Em algumas dessas áreas, as taxas brutas de mortalidade neonatal foram semelhantes à média da região central e ocidental da África (em torno de 45/1.000 nascidos vivos, em 2004), onde se concentram os maiores índices desta mortalidade, no mundo 13 . 
Como se sabe, a gravidez na adolescência tem sido associada a resultados adversos ao recémnascido, seja por questões biológicas, baixos níveis de escolaridade ou outros fatores que levam, por exemplo, ao pré-natal inadequado ${ }^{9,23}$. Igualmente, elevadas proporções de nascidos vivos de baixo peso estão associadas, em geral, a baixos níveis de desenvolvimento socioeconômico e de assistência materno-infantil 24,25,26. Todavia, estudos mais recentes demonstram um aumento de baixo peso ao nascer em grupos populacionais de maior renda, talvez associado a interrupções da gravidez 27, entre outras possíveis causas, situação que configura o chamado paradoxo do baixo peso 28 .

Embora tenham sido encontradas em algumas zonas do centro da cidade taxas médias brutas que variaram entre 6,8/1.000 nascidos vivos e 14,2/1.000 nascidos vivos e, portanto, com algumas ZI apresentando taxas inferiores à media brasileira (12,3/1.000 nascidos vivos) e a de Salvador (17,4/1.000 nascidos vivos) no mesmo período, baixos valores predominaram naquelas ZI situadas ao sul e leste que coincidem com as áreas nas quais prevaleceram os nascimentos vivos de mães com idade igual ou superior a 35 anos e de parto cesáreo. Segundo dados do Censo Demográfico 2000 (IBGE. http://www.ibge.gov.br/cida desat/default.php, acessado em 10/Nov/2007), essas áreas em sua maioria apresentavam baixos percentuais de chefes de família com renda igual ou inferior a dois salários mínimos e de moradias do tipo favela, e altos percentuais de escolarização de mulheres em idade fértil e de domicílios com abastecimento de água da rede geral. Essa situação está em consonância com os resultados de estudos que referem que altos índices desse tipo de parto e de mães com idades mais elevadas, com frequência, estão relacionados às camadas sociais mais abastadas 29,30. Pode-se considerar ainda, que a adequada situação socioeconômica dessas zonas de residência de Salvador, possivelmente, também proporcionou melhor acesso aos serviços e informações de saúde aos residentes nessas áreas, e assim influenciaram positivamente no cuidado à saúde infantil. Chama-se aqui a atenção para as áreas de transição de baixo/alto risco, por merecerem monitoramento especial, na medida em que podem sofrer a influência de fatores de risco de áreas vizinhas e aumentarem a mortalidade de menores de 28 dias.

Não pode deixar de ser comentada a homogeneidade na distribuição da proporção de nascidos vivos prematuros nos espaços intraurbanos de Salvador, de modo que não foi possível traçar um nítido padrão relacionado às condições socioeconômicas das áreas, fato também observado em investigação abrangendo os bairros do
Rio de Janeiro ${ }^{31}$. Contudo, foram identificados maiores percentuais em área deteriorada da cidade formada por zonas de altas taxas de mortalidade neonatal, o que condiz com resultados de outras investigações 25,32.

Embora a superposição espacial entre a mortalidade neonatal e os fatores de risco selecionados, constatada com base na respectiva representação gráfica, tenha sido confirmada pela análise bivariada de Moran, estas relações perderam a significância quando os fatores foram avaliados conjuntamente, à exceção da "proporção de nascidos vivos com baixo peso", que apresentou a maior contribuição para o aumento das taxas de mortalidade neonatal nesta investigação, sendo considerada a principal responsável pela determinação do padrão espacial da mortalidade neonatal em Salvador, no período do estudo. De 2000-2006, cerca de $10 \%$ a $11 \%$ dos nascidos vivos nessa capital apresentaram baixo peso (http:// w3.datasus.gov/datasus/datasus.php, acessado em 17/Jul/2009), portanto, em proporção superior à média brasileira de 8\% (de 1999-2006), da América Latina e do Caribe (9\%) e dos países industrializados $(7 \%)$, porém inferior à proporção mundial, a de países africanos (15\%) e também dos países do sul asiático (29\%), no mesmo período 33 . No presente estudo, valores superiores a $10 \%$ foram encontrados em $59,1 \%$ das ZI de Salvador, e são considerados inaceitáveis, conforme convenções internacionais ${ }^{34}$. Examinando-se as causas de óbitos neonatais em Salvador, nota-se que "transtornos não especificados relacionados à prematuridade e baixo peso", a partir de 2002, estavam entre as três principais causas de óbitos neonatais, e se posicionaram em primeiro lugar em 2005 e 2006 (http://w3.datasus.gov/ datasus/datasus.php, acessado em 17/Jul/2009), evidenciando que esta condição de nascimento vem desempenhando um papel importante na determinação da mortalidade nos primeiros 28 dias de vida.

Outros estudos com dados agregados e abordagem espacial também demonstraram a participação do baixo peso ao nascer nesse processo $7,8,23$, relacionando-o a áreas com piores condições de vida ${ }^{32}$, embora esta relação nem sempre seja comprovada, especialmente quando se trata de estudos de agregados espaciais, configurando o baixo peso ao nascer mais como um eventosentinela do óbito infantil e menos um indicador de risco agregado 35. Em função do alto potencial de evitabilidade do baixo peso ao nascer e da prematuridade, é oportuno ressaltar a necessidade de adoção de medidas que visem a melhorias no atendimento pré-natal e no nascimento, no sentido de desenvolver ações mais eficazes para o diagnóstico e intervenção nestes e sobre outros 
fatores de risco para o óbito neonatal. Todavia, deve-se também atentar para o fato de que esses fatores de risco são os mediadores por meio dos quais agem outros determinantes, a exemplo do nível socioeconômico 17 .

Salienta-se que alguma cautela deve ser adotada quando da interpretação dos resultados aqui apresentados. Por se basear em dados secundários, os achados deste estudo podem ter sido afetados por sua cobertura e qualidade, embora tratando-se de dados provenientes de uma capital, onde se espera que os sistemas de informações sejam mais desenvolvidos, as informações mais fidedignas e um nível mínimo de sub-registro de óbitos e nascimentos, caso este ocorra. Por sua vez, nada se pode afirmar sobre a possibilidade de maior sub-registro de óbitos em áreas mais pobres, além da maior propensão de classificação errônea do óbito neonatal precoce como óbito fetal, conforme já destacado em outras publicações 12,36,37, visto que a qualidade dos registros também pode ser afetada pelas diferenças entre os grupos, oriundas da desigualdade social e econômica 37 . Também chama-se a atenção para o fato de se tratar de um estudo de agregados, no qual a unidade espacial de análise utilizada (no caso, ZI) representa divisões geográfico-administrativas pré-definidas, e não exatamente áreas mais homogêneas quanto às condições socioeconômicas.

Embora a pequena densidade demográfica de algumas dessas áreas pudessem distorcer o valor da medida de efeito, foram calculadas taxas médias de mortalidade neonatal para o período 2000-2006 e, além disto, as mesmas foram submetidas a uma técnica estatística para minimizar este problema, como referido na seção dos métodos. Cabe destacar ainda, que a configuração da distribuição espacial da proporção de nascidos vivos de mães com pré-natal inadequado à idade gestacional pode ter sido influenciada pela categorização do número de consultas pré-natais previamente definido na Declaração de Nascidos Vivos, o que dificultou estabelecer a inadequação do número destas consultas com maior precisão.

A despeito dessas possíveis limitações, podese concluir que não foi aleatória a distribuição espacial da mortalidade neonatal em Salvador, entre 2000 e 2006. Apesar de, atualmente, não se dispor de outros estudos de análise espacial da mortalidade neonatal nessa capital que possibilitem fazer comparação, constata-se que a distribuição ora apresentada é muito semelhante à da mortalidade infantil nesse mesmo município, nas décadas de 1980 e 1990 36,38. Se por um lado, o baixo peso ao nascer foi considerado como o mais importante fator explicativo da variação espacial da mortalidade neonatal, por outro, os diferenciais observados nas taxas desta mortalidade, entre as diversas áreas da cidade, demonstram que a existência das desigualdades sociais, expressa pelas condições de vida da população, pode estar contribuindo de forma decisiva para o padrão espacial detectado. Por fim, depreende-se que a aplicação de técnicas de análises espaciais mostrou-se bastante útil na identificação de grupos mais vulneráveis e, portanto, o seu emprego pelos profissionais responsáveis pela vigilância de óbitos infantis também deve ser estimulado.

Considerando os achados deste estudo, não restam dúvidas sobre a necessidade da adoção de medidas voltadas à atenção à saúde maternoinfantil no que se refere à prevenção do baixo peso ao nascer, particularmente, nas áreas de maior risco de morte neonatal. Aliado a isso, torna-se imperativo o estabelecimento de políticas públicas com ações mais abrangentes visando à melhoria das condições de vida da população. 


\section{Resumo}

Realizou-se estudo de agregados espaciais visando a identificar padrões na distribuição espacial da mortalidade neonatal, bem como fatores associados, em Salvador, Bahia, Brasil, 2000-2006. Foram construídos mapas temáticos e usadas técnicas para apreciação formal de dependência espacial. Mediante modelos de regressão linear múltipla (espacial e não espacial) verificou-se a relação entre distribuição espacial dessa mortalidade e fatores selecionados. Evidenciou-se autocorrelação espacial para a mortalidade neonatal $(I=0,17 ; p=0,0100)$, não havendo, portanto, aleatoriedade em sua distribuição. Foi delineado um padrão espacial em que os maiores riscos (> 9,0/1.000 nascidos vivos) concentraram-se em áreas do centro e subúrbio, onde reside a população de menor condição socioeconômica, mostrando-se esta distribuição associada aos fatores de risco analisados. A proporção de nascidos vivos com baixo peso foi a única variável significativamente associada à mortalidade neonatal. Possivelmente, as condições de vida da população contribuíram para a desigual distribuição espacial da mortalidade neonatal nesse município.

Análise Espacial; Mortalidade Neonatal; Zona de Risco; Fatores de Risco

\section{Colaboradores}

A. C. Gonçalves, M. C. N. Costa e J. U. Braga contribuíram na concepção e execução do projeto, análise e interpretação dos dados, redação do artigo, revisão crítica relevante do conteúdo intelectual, aprovação da versão final.

\section{Referências}

1. Santos M. Espaço e método. 3a Ed. São Paulo: Nobel; 1992.

2. Costa MC, Teixeira MG. Concepção de "espaço" na investigação epidemiológica. Cad Saúde Pública 1999; 15:271-9.

3. Loyola E, Castillo-Salgado C, Nájera-Aguilar P, Vidaurre M, Mujica OJ, Martinez-Piedra R. Geographic information systems as a tool for monitoring health inequalities. Rev Panam Salud Pública 2002; 12:415-28.

4. Álvarez G, Lara F, Harlow SD, Denman C. Mortalidad infantil y marginación urbana: análisis espacial de su relación en una ciudad de tamaño medio del noroeste mexicano. Rev Panam Salud Pública 2009; 26:31-8.
5. Grady SC, Enander H. Geographic analysis of low birthweight and infant mortality in Michigan using automated zoning methodology. Int J Health Geogr 2009; 8:10.

6. Morais Neto OL, Barros MB, Martelli CM, Silva AS, Cavenaghi SM, Siqueira Junior JB. Diferenças no padrão de ocorrência da mortalidade neonatal e pós-neonatal no Município de Goiânia, Brasil, 1992-1996: análise espacial para identificação das áreas de risco. Cad Saúde Pública 2001; 17: 1241-50.

7. Shimakura SE, Carvalho MS, Aerts DR, Flores R. Distribuição espacial do risco: modelagem da mortalidade infantil em Porto Alegre, Rio Grande do Sul, Brasil. Cad Saúde Pública 2001; 17:1251-61. 
8. Bezerra Filho JG, Kerr LR, Miná DL, Barreto ML. Distribuição espacial da taxa de mortalidade infantil e principais determinantes no Ceará, Brasil, no período 2000-2002. Cad Saúde Pública 2007; 23:1173-85.

9. Andrade CL, Szwarcwald CL. Análise espacial da mortalidade neonatal precoce do Município do Rio de Janeiro 1995-1996. Cad Saúde Pública 2001; 17:1199-210.

10. Andrade SM, Soares DA, Matsuo T, Souza RK, Mathias TA, Iwakura ML, et al. Condições de vida e mortalidade infantil no Estado do Paraná, Brasil, 1997/2001. Cad Saúde Pública 2006; 22:181-9.

11. Nascimento LF, Batista GT, Dias NW, Catelani CS, Becker D, Rodrigues L. Análise espacial da mortalidade neonatal no Vale do Paraíba, 1999 a 2001. Rev Saúde Pública 2007; 41:94-100.

12. Leal MC, Szwarcwald CL. Características da mortalidade neonatal no Estado do Rio de Janeiro na década de 80: uma visão espaço temporal. Rev Saúde Pública 1997; 31:457-65.

13. World Health Organization. Neonatal and perinatal estimatives. Country, regional and global estimatives 2004. Geneva: World Health Organization; 2007.

14. Martins EF, Velásquez-Melandéz G. Determinantes da mortalidade neonatal a partir de uma coorte de nascidos vivos, Montes Claros, MG, 1997-1999. Rev Bras Saúde Matern Infant 2004; 4:405-12.

15. Secretaria Municipal de Saúde de Salvador. Dados SIM/SINASC (CD-ROM). Salvador: Diretoria de Informação em Saúde, Secretaria Municipal de Saúde de Salvador; 2007.

16. Ministério da Saúde. Pré-natal e puerpério: atenção qualificada e humanizada - manual técnico. Brasília: Ministério da Saúde; 2006.

17. Paulucci RS, Nascimento LF. Mortalidade neonatal em Taubaté: um estudo caso-controle. Rev Paul Pediatr 2007; 25:358-63.

18. Cressie N, Read, TR. Spatial data analysis of regional counts. Biom J 1989; 31:699-719.

19. Cliff AD, Ord JK. Spatial process: models and applications. London: Pion; 1981.

20. Paim JS, Silva LM, Costa MC, Prata PR, Lessa I. Desigualdades na situação de saúde do município de Salvador e relações com as condições de vida. Rev Ciênc Méd Biol 2003; 2:30-9.

21. Andrade AB, Brandão, PR. Geografia de Salvador. Salvador: EDUFBA; 2006.

22. Carvalho IM, Pereira GC. Como anda Salvador e sua região metropolitana. Salvador: EDUFBA; 2006.

23. Minuci EG, Almeida MF. Diferenciais intra-urbanos de peso ao nascer no município de São Paulo. Rev Saúde Pública 2009; 43:256-66.

24. Rede Interagencial de Informações para Saúde. Informe de situação e tendências: demografia e saúde. Brasília: Organização Pan-Americana da Saúde; 2009.

25. Gurgel RQ, Dias IM, França VL, Castañeda DF. Distribuição espacial do baixo peso ao nascer em Sergipe, Brasil, 1995/1998. Cad Saúde Pública 2005; 21:1329-37.
26. Andrade CL, Szwarcwald CL, Gama SG, Leal MC. Desigualdades sócio-econômicas do baixo peso ao nascer e da mortalidade perinatal no Município do Rio de Janeiro, 2001. Cad Saúde Pública 2004; 20 Suppl 1:S44-51

27. Barros FC, Victora CG, Matijasevich A, Santos IS, Horta BL, Silveira MF, et al. Preterm births, low birthweight, and intra-uterine growth restriction in three birth cohorts in southern Brazil. 1982, 1993 and 2004. Cad Saúde Pública 2008; 24 Suppl 3:S390-8.

28. Silva AA, Bettiol H, Barbieri MA, Pereira MM, Brito LG, Ribeiro VS, et al. Why are the low birthweight rates in Brazil higher in richer than in poorer municipalities? Exploring the epidemiological paradox of low birthweight. Paediatr Perinat Epidemiol 2005; 19:43-9.

29. Althabe F, Sosa C, Belizán JM, Gibbons L, Jacquerioz F, Bergel E. Cesarean section rates and maternal and neonatal mortality in low, medium, and highincome countries: an ecological study. Birth 2006; 33:270-7.

30. Ronsmans C, Holtz S, Stanton C. Socioeconomic differentials in caesarean rates in developing countries: a retrospective analysis. Lancet 2006; 368:1516-23.

31. D'Orsi E, Carvalho MS, Cruz OG. Similarity between neonatal profile and socioeconomic index: a spatial approach. Cad Saúde Pública 2005; 21:786-94.

32. Luo ZC, Kierans WJ, Wilkins R, Liston RM, Mohamed J, Kramer MS, et al. Disparities in birth outcomes by neighborhoods income: temporal trends in rural and urban areas, British Columbia. Epidemiology 2004; 15:679-86.

33. Fundo das Nações Unidas para a Infância. Situação mundial da infância 2008. Sobrevivência infantil. Brasília: Fundo das Nações Unidas para a Infância; 2007.

34. Organização das Nações Unidas. Declaração mundial sobre a sobrevivência, a proteção e o desenvolvimento da criança. New York: Organização das Nações Unidas; 1990.

35. d'Orsi E, Carvalho MS. Perfil de nascimentos no Município do Rio de Janeiro: uma análise espacial. Cad Saúde Pública 1998; 14:367-79.

36. Costa MC, Azi PA, Paim JS, Silva LM. Mortalidade infantil e condições de vida: a reprodução das desigualdades sociais em saúde na década de 90. Cad Saúde Pública 2001; 17:555-67.

37. Campos D, Loschi RH, França E. Mortalidade neonatal precoce hospitalar em Minas Gerais: associação com variáveis assistenciais e a questão da subnotificação. Rev Bras Epidemiol 2007; 10:223-38.

38. Paim JS, Costa MC, Cabral V, Mota IA, Neves RB. Spatial distribuition of proportional infant mortality and certain socioeconomic variables in Salvador, Bahia, Brazil. Bull Pan Am Health Organ 1987; 21:225-39.

Recebido em 29/Dez/2010

Versão final reapresentada em 08/Abr/2011 Aprovado em 06/Mai/2011 